This item was submitted to Loughborough's Research Repository by the author.

Items in Figshare are protected by copyright, with all rights reserved, unless otherwise indicated.

\title{
Logistics of using the Actiheart physical activity monitors in urban Mexico among 7- to 9-year-old children
}

PLEASE CITE THE PUBLISHED VERSION

http://dx.doi.org/10.1002/ajhb.21150

\section{PUBLISHER}

(C) 2011 Wiley-Liss, Inc.

\section{VERSION}

NA (Not Applicable or Unknown)

\section{PUBLISHER STATEMENT}

This work is made available according to the conditions of the Creative Commons Attribution-NonCommercialNoDerivatives 4.0 International (CC BY-NC-ND 4.0) licence. Full details of this licence are available at: https://creativecommons.org/licenses/by-nc-nd/4.0/

\section{LICENCE}

CC BY-NC-ND 4.0

\section{REPOSITORY RECORD}

Wilson, Hannah J., Federico Dickinson, Paula L. Griffiths, Barry Bogin, and Maria Ines Varela Silva. 2019. "Logistics of Using the Actiheart Physical Activity Monitors in Urban Mexico Among 7- to 9-year-old Children". figshare. https://hdl.handle.net/2134/19374. 


\title{
Short Report
}

\section{Logistics of Using the Actiheart Physical Activity Monitors in Urban Mexico Among 7- to 9-Year-Old Children}

\author{
HANNAH WILSON, ${ }^{1 *}$ FEDERICO DICKINSON,${ }^{2}$ PAULA GRIFFITHS, ${ }^{1}$ BARRY BOGIN, ${ }^{1}$ AND MARIA INÊS VARELA-SILVA ${ }^{1}$ \\ ${ }^{1}$ Centre for Global Health and Human Development, School of Sport, Exercise and Health Sciences, Loughborough University, \\ Loughborough LE11 3TU, United Kingdom \\ ${ }^{2}$ Departamento de Ecología Humana, Centro de Investigación y de Estudios Avanzados del Instituto Politécnico Nacional \\ (Cinvestav)-Unidad Mérida, Mérida 97310, Yucatán, México
}

\begin{abstract}
Logistics of using new measurement devices are important to understand when developing protocols. This paper discusses the logistics of using Actiheart physical activity monitors on children in an urban, tropical environment in a developing country. Actiheart monitoring of 36 children aged 7-9 years old was undertaken for 7 days in the city of Mérida, Yucatán, Mexico. The Actiheart proved fragile for children and difficult to mend in the field. The excessive sweating due to the tropical climate caused poor adherence of the electrode pads, requiring a pad change midway through and extra pads to be provided. Also extra time was needed to be allotted for increased instructions to participants and their mothers and for individual calibration. When collecting objectively measured physical activity data under harsh conditions, the protocol must accommodate local conditions and device limitations and allow increased time with participants to obtain good quality data. Am. J. Hum. Biol. 23:426-428, $2011 . \quad$ (c) 2011 Wiley-Liss, Inc.
\end{abstract}

Researchers need to be informed about the feasibility and logistics of using measurement devices to perform the most efficient research possible. This short report discusses the logistics of using the Actiheart to estimate levels of physical activity and energy expenditure, under free living conditions, among children in urban Mexico. The aim is to inform other researchers planning to use the device in similar field settings of some of the challenges our research team has found, particularly among children in an urban tropical environment in a developing country.

Physical activity patterns are changing worldwide and the developing world is lacking the physical activity data needed to fully understand their activity patterns and effectively combat the rise in overweight and obesity (Satia, 2010). Objectively measured physical activity is more accurate than self-reported data (Westerterp, 2009). The Actiheart provides a very high quality method of estimating physical activity in humans. It is a combined movement sensor and heart rate monitor (Brage et al., 2004), and is composed of two sensors connected by a short wire, which is clamped to the ECG electrode pads placed on the chest of the participants. It combines a uniaxial accelerometer with a heart rate monitor to provide a more accurate estimation of physical activity levels and energy expenditure than either method individually (Barreira et al., 2009; Corder et al., 2005). The Actiheart has been validated in free living conditions in adults in developing country (Assah et al., 2010) and Western European children (Corder et al., 2007, de Bock et al., 2010). These validation studies show that the Actiheart is an appropriate tool for the assessment of physical activity in free-living populations.

\section{METHODS AND RESULTS}

The researchers used 11 Actihearts to monitor 36 children (18 male) aged 7 to 9 years of low SES; for descriptives, see Table 1 . The monitoring of each child took place over 1 week in the tropical, urban environment of Mérida, Yucatán, Mexico, during the dry and rainy seasons (April to July) of 2010. Ethical clearance was obtained from Loughborough University and the Bioethical Committee of Cinvestav in Mexico. Informed consent was obtained from each mother and assent obtained from each child.

Over the course of data collection, complete 5-day physical activity data were successfully collected for 20 of the 36 children. The average total energy expenditure was $1975 \mathrm{kcal} /$ day (288 SD) with no difference between weekdays and weekends or sexes. For the 16 cases with incomplete data: in 10 cases, Actihearts fell off for substantial periods; and in six cases, heart rate data were not consistently recorded. Most of the Actihearts fell off before the protocol was amended to include greater information and instructions to the mothers. Accelerometry data was collected consistently when the Actiheart was worn. The unreliable heart rate data was due to poor adherence of the electrode pads to the skin. Also, six of 11 Actihearts broke and were not able to be fixed during fieldwork. The wires were disconnected in two devices during shirtless rough play, and the lids detached in four devices. This is higher than the failure rate of eight of 24 while monitoring 13-year olds for 5 days in Brazil reported by Reichert et al., (2009). The lid detachments did not vary with age, sex, physical activity, or SES of the child. Two Actihearts were shipped to the U.K. for repair and were confiscated

Contract grant sponsor: Wenner-Gren Foundation; Contract grant number: ICRG-93.

*Correspondence to: Hannah Wilson, Centre for Global Health and Human Development, School of Sport, Exercise and Health Sciences, Loughborough University, Loughborough LE11 3TU, United Kingdom.

E-mail: H.Wilson@lboro.ac.uk

Received 7 September 2010; Revision received 13 December 2010; Accepted 15 December 2010

DOI 10.1002/ajhb.21150

Published online 29 March 2011 in Wiley Online Library (wileyonlinelibrary. com). 
TABLE 1. Descriptives statistics for urban Maya children ages 7 to 9 years

\begin{tabular}{|c|c|c|c|}
\hline & Girls & Boys & Total \\
\hline & Mean (SD) & Mean (SD) & Mean (SD) \\
\hline$N$ & 18 & 18 & 36 \\
\hline Height (cm) & $122.12(7.86)$ & $120.28(5.79)$ & $121.16(6.75)$ \\
\hline Height-for-age $z$-score ${ }^{\mathrm{b}}$ & $-1.20(0.89)$ & $-1.31(0.64)$ & $-1.15(0.86)$ \\
\hline Weight $(\mathrm{kg})$ & $29.38(8.72)$ & $25.15(4.38)$ & $27.04(6.91)$ \\
\hline Weight-for-age $z$-score ${ }^{\mathrm{b}}$ & $-0.05(1.00)^{\mathrm{a}}$ & $-0.71(0.65)^{\mathrm{a}}$ & $-0.45(0.88)$ \\
\hline Body mass index $\left(\mathrm{kg} / \mathrm{ht}^{2}\right)$ & $19.38(4.18)$ & $17.25(2.14)$ & $18.20(3.34)$ \\
\hline Body mass index for-age $z$-score $\left(\mathrm{kg} / \mathrm{ht}^{2}\right)^{\mathrm{b}}$ & $0.88(1.13)$ & $0.50(0.81)$ & $0.56(0.95)$ \\
\hline Total energy expenditure $(\mathrm{kcal})^{\mathrm{c}}$ & $1987.1(395.2)$ & $1986.0(282.2)$ & $1986.5(332.6)$ \\
\hline
\end{tabular}

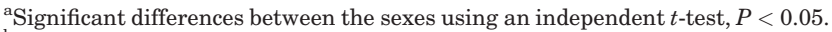

${ }^{b}$ Sex- and age-specific $z$-scores calculated using Frisancho's Comprehensive reference (2008).

'Total energy expenditure calculations made using the Actiheart equations for children using group reference data.

by Mexican customs who demanded three times the cost of an Actiheart for their release.

\section{DISCUSSION}

The Actiheart is relatively fragile for long term, free living monitoring of children. Two of the children disconnected the wires of their Actihearts while playing. Only the manufacturer was able to reconnect the wire and the monitor, due to the nature of the connection, i.e., clamped not soldered. The two could have been reconnected by soldering in the field, which would have resulted in permanent damage to the Actiheart. Therefore, it was necessary to ship the Actihearts back to the UK. Import laws caused customs delays in the U.K. and Mexico, so the broken devices were unavailable for the rest of fieldwork. Rough playing of the children, especially without shirts, can lead to wire disconnection.

Not all of the problems were associated with the children. Four lids detached exposing the electronics due to the high humidity, which reduced the adhesiveness of the glue. Reattachment had to occur in the U.K. The tropical climate also caused poor adherence of the electrode pads to the skin. The Actihearts were developed in the U.K., where extremely high temperatures are unusual. Our fieldwork was conducted in Yucatán, Mexico, where the average temperature was $33.0^{\circ} \mathrm{C}$ and with $60 \%$ humidity in the dry season, in a poor population without access to air conditioning. Thus, the levels of sweating were very high with slow moisture evaporation. The electrode pads did not stay reliably adhered to the chest for more than 3 days. The lack of long-term adherence was also encountered when using Actihearts in adults in sub-Saharan African (Assah et al., 2010). Therefore, the protocol was amended for researchers to provide the mothers with extra pads and give revamped instructions on how to attach them. Also, the researchers changed the pads midway through the week long monitoring. Approximately $25 \%$ of the pads required changing outside of the researchers' visits. Therefore, a minimum of six pads were required for each participant for the week long monitoring. Even when the pads were attached to the chest, the Actiheart did not always maintain close enough contact with the skin for constant heart rate monitoring. When this occurred for short periods $(<5 \mathrm{~min})$, the software was able to perform interpolation to estimate the heart rate of the lost period (CamNtech, 2009). This issue was minimized by the frequent changing of electrode pads and increased instructions to the mothers. This protocol change decreased the amount of lost data substantially. Overall, ECG electrode pads do not stay consistently adhered in free living conditions in hot climates. Researchers working in such environments should budget for extra electrode pads and have a protocol that includes multiple participant visits.

The children were too young to follow the instructions given at the beginning of the monitoring period. Gaining access to mothers to provide instructions on the day the children started monitoring was challenging but often possible. Actihearts were generally placed on the children at school, so in these cases, mothers either came to the school or the fieldworkers went to the child's house to find the mother or caregiver. The mothers were provided with a package containing an instruction sheet and extra electrode pads. The instruction sheet included what to do if the Actiheart or a pad came off, how to attach the Actiheart to the pad and the researchers' phone numbers. At this time, the children were asked not to play rough team sports, to prevent damage to the Actiheart. This request to avoid these behaviors could have changed activity patterns, leading to a less accurate estimation of habitual physical activity in the children. After these instructions were added to the protocol, no more wires were disconnected. Giving instructions to each mother increased the time requirements on both mothers and fieldworkers but also greatly increased the quality of the data.

Physical activity measurement in children is challenging, particularly when the measurement device is relatively fragile and worn continuously in free-living conditions. In our sample, these problems were compounded by the climate. The lack of adherence of the electrode pads in tropical climates will occur no matter the age of the participant but is more likely to be quickly fixed by an adult than a child. Therefore, the data quality is likely to be higher in adults. A factor that must be considered that is inherent to objective heart rate monitoring is individual calibration. Interindividual variations in heart rates are large and must be controlled for effective physical activity estimations (Westerterp 2009). Reference curves can be used but increase the error of estimation (ibid). For this study, individual calibration was done using the walking exercise test and 10-15 min was required per test in this study. The Actiheart software allows multiple participants to perform the calibration test simultaneously, thereby, reducing the overall time requirements of individual calibration. Two children were able to do the calibration simultaneously quite easily. Individual calibration is important for accurate results and is easily done, and the time involved must be considered in logistical plans. 


\section{CONCLUSION}

The use of the Actiheart for long-term monitoring in free-living children is logistically challenging and time consuming. The difficulties in measuring physical activity in children were compounded when using them in poor communities without air conditioning in hot climates. On the basis of our experience, we recommend the following: (1) the Actiheart needs to be more durable or more easily repaired outside of the UK and (2) new ECG electrode pads are needed that can reliably adhere under conditions of consistent sweating before Actihearts can be widely and easily used in free-living populations.

These logistical challenges do not eliminate the possibility of using the Actiheart in such environments but should be considered during the planning of field studies. The Actiheart provides high quality data and can be used under harsh conditions with the proper protocols, though breakages should be expected.

\section{ACKNOWLEDGMENT}

The authors thank to the wonderful women and children who participated; to the indispensible fieldworkers Adriana Vázquez Vázquez, Jenice Tut Be, and Hugo Azcorra; engineers Felipe Kantun and Gaspar Euan for advice and assistance repairing the Actihearts; Officers of the Department of Education of the Yucatan Government and the school directors for allowing our work.

\section{Literature Cited}

Assah FK, Ekelund U, Brage S, Wright A, MBanya JC, Wareham NJ. 2010. Accuracy and validity of a combined heart rate and motion sensor for the measurement of free-living physical activity energy expenditure in adults in Cameroon. Int J Epidemiol. Published ahead of print: DOI: 10.1093/ije/dyq098.

Barreira TV, Kang M, Caputo JL, Farley RS, Renfrow MS. 2009. Validation of the Actiheart monitor for the measurement of physical activity. Int $\mathrm{J}$ Exer Sci 2:60-71.

Brage S, N Brage, PW Franks, U Ekelund, NJ Wareham. 2005. Reliability and validity of the combined heart rate and movement sensor Actiheart. Eur J Clin Nutr 59:561-570.

CamNtech. 2009. The Actiheart User Manual. Issue version: 4.0.34. Cambridge: CamNtech Ltd.

Corder K, Brage S, Mattocks C, Ness A, Riddoch C, Wareham NJ, Ekelund U. 2007. Comparison of two methods to assess PAEE during six activities in children. Med Sci Sport Exer 39:2180-2188.

Corder K, Brage S, Wareham NJ, Ekelund U. 2005. Comparison of PAEE from combined and separate heart rate and movement models in children. Med Sci Sport Exer 37:1761-1767.

De Bock F, J Menze, S Becker, D Litaker, J Fischer, I Seidel, 2010. Combing accelerometry and heart rate for assessing preschoolers' physical activity. Med Sci Sport Exer 42:2237-2243.

Fossati Reichert F, Batista Menezes AM, Wells JC, Ekelund U, Machado Rodrigues F, Curi Hallal P. 2009. A methodological model for collecting high-quality data on physical activity in developing settings-the experience of the 1993 Pelotas (Brazil) Birth Cohort Study. J Phys Activ Health 6:360-366.

Frisancho AR. 2008. Anthropometric standards: an interactive nutritional reference of body size and body composition for children and adults. Ann Arbor, Michigan: University of Michigan Press. 335 p.

Satia JA. 2010. Dietary acculturation and the nutrition transition: an overview. Appl Physiol Nutr Metab 35:219-223.

Westerterp KR. 2009. Assessment of physical activity: a critical appraisal. Eur J Appl Physiol 105:823-828. 